\title{
Oedipe et les fouille-merde
}

Qu'est-ce que le journalisme d'investigation?

Oedipe and the muck-rakers.

\section{Xavier Delcourt}

\section{(2) OpenEdition}

Journals

Édition électronique

URL : http://journals.openedition.org/edc/2678

DOI : $10.4000 /$ edc. 2678

ISSN : 2101-0366

Éditeur

Université Lille-3

\section{Édition imprimée}

Date de publication : 1 mai 1994

Pagination : 25-43

ISSN : 1270-6841

\section{Référence électronique}

Xavier Delcourt, "Oedipe et les fouille-merde », Études de communication [En ligne], 15 | 1994, mis en ligne le 26 décembre 2011, consulté le 20 avril 2019. URL : http://journals.openedition.org/edc/2678 DOI : $10.4000 /$ edc. 2678

Ce document a été généré automatiquement le 20 avril 2019.

(c) Tous droits réservés 


\title{
Oedipe et les fouille-merde
}

\author{
Qu'est-ce que le journalisme d'investigation?
}

Oedipe and the muck-rakers.

\section{Xavier Delcourt}

1 Depuis une dizaine d'années, on célèbre l'apparition sur le continent d'un genre journalistique nouveau : l'investigation. Désormais installé en France, en Espagne, en Italie, dans les pays nordiques, il se propagerait aujourd'hui jusqu'en Russie. Nouveau genre?

2 Le terme investigation est attesté en français depuis le XIII ${ }^{\mathrm{e}}$ siècle. Mais dans son acception journalistique actuelle, il sonne sans équivoque comme un américanisme récemment importé (investigative reporting). Le journalisme d'investigation serait un genre américain ou anglo-américain - mis en lumière et symbolisé par le fameux «Watergate ».

3 Pourtant, les revendications d'antériorité, les dénonciations excédées d'un effet de mode ne manquent pas, jusque dans les rangs des journalistes. Sommairement, elles soulignent au moins deux redondances:

4 - La matière traitée n'est pas tout à fait inédite. Les malversations des puissants de la politique, de la finance, de l'économie font depuis longtemps l'objet de dénonciations publiques ou clandestines : théâtre, pamphlets, chansons, satires, et toute une littérature d'espionnage et d'essais sur le gouvernement des hommes.

5 - Le support même n'est pas insolite: la presse militante, au XIX siècle, la presse à scandale du début $\mathrm{du} \mathrm{XX}^{\mathrm{e}}$, le Canard enchainé depuis seize lustres, à des degrés de spécialisation divers, se sont emparés de l'exercice immoral ou délictueux du pouvoir.

6 A ces objections, le journalisme d'investigation fourbit ses réponses, énigmatiques et désarmantes. Il prétend avant tout se distinguer par des procédures d'établissement et des procédés d'exposition des faits ; par un temps de travail préparatoire qui n'obéit pas au rythme de l'actualité, ni à l'économie traditionnelle des entreprises d'information ; par un agencement des témoignages qui suit une progression calculée; par tout un système de garde-fous, faisant intervenir des savoirs juridiques. Soit. Mais de cette démarche journalistique, il n'existe pas, en France, de description. Quels sont les traits distinctifs de 
« l'investigation »? Où trouve-t-elle ses instruments ? Dans quelles lignées les puise-t-elle ? A quel domaine de pratiques les applique-t-elle?

\section{Ordres de vérité.}

7 L'information journalistique joue sur une série de registres d'exposition qui obéissent à des règles de validation incertaines. Celles, par exemple, qui sont supposées régir « l'objectivité » d'un reportage, ou la " fidélité » d'un compte rendu. On n'ignore pas les difficultés que soulève la formulation de ces règles. Elles comptent pour beaucoup dans l'appréhension du journalisme comme simple véhicule de normes et dans son incapacité à se hisser au rang d'acte de savoir. Elles entretiennent enfin une confusion prolixe entre liberté de la presse et liberté d'opinion.

8 Pourtant, la difficulté d'énoncer des régles tient peut-être en partie à l'imprécision des concepts - et donc des pratiques - qu'elles sont censées décrire. Plutôt que de recourir à cet arsenal hétéroclite, on gagnerait sans doute à distinguer deux ordres de vérité ${ }^{1}$, qui s'exercent concurremment à l'intérieur des médias (et plus généralement du savoir occidental) : la vérité de circonstances et la vérité de constat. Elles recourent l'une et l'autre à des technologies distinctes de production du vrai qui disposent de règles de validation énonçables.

9 Le premier ordre de technologies, emblématisé par l'alchimie, ce grand modèle du savoir médiéval, se démarquerait radicalement du discours scientifique. Il repose sur l'idée que la vérité a ses instants favorables, ses lieux privilégiés pour être non seulement exposée mais produite. Vérité-événement, sa liturgie implique un calendrier des occasions, une géographie des « sites », un savoir des rituels qui tracent les arcanes de son invocation.

10 Toute une série de registres journalistiques, de la rubrique astrologique au compte rendu sportif, en passant par l'éditorial ou l'épreuve du « face à face » peuvent-être rangés sous cet ordre.

11 La vérité de constat, elle, prétend s'inscrire dans un second ordre technologique : celui qui s'attache avant tout, quel que soit le moment, à trouver sur n'importe quel point la bonne perspective, l'angle convenable, les instruments appropriés.

12 Alors que les vérités du premier ordre ne s'obtiennent qu'au terme d'épreuves initiatiques, que leur transmission, leur reproduction requièrent des individus d'exception, celles du second ordre trouvent leur validation dans la forme de la connaissance expérimentale : elles sont en droit reconnaissables et reproductibles par tous.

13 L'investigation se doit d'appartenir à ce second ordre puisqu'elle a pour finalité affichée la poursuite et la manifestation d'une vérité commune, accessible au jugement de chacun. A ce titre elle s'inscrit dans une (ou des) famille(s) d'énoncés soumis à des règles de recevabilité particulières. Elle est tenue, comme acte de formulation, de passer le test d'une police discursive qui sélectionne le véridique : le test du « vrai » ou du « faux ». Sur quelle lignée perspective l'inscrive, de quel département de la police du vrai relève-t-elle ? 


\section{L'invention de l'enquête.}

14 De la vérité de circonstances, avec ses rituels d'entraînement de soi, ses moments d'épreuve, on avance parfois qu'elle serait vieille comme l'humanité. Fonds magicoreligieux qui plongerait dans la nuit des temps habités, sorte de soupe primitive des instincts, qui persisterait jusque dans les inclinaisons inavouables des plus savants. Peutêtre la vérité de constat n'en est-elle, en effet, qu'une forme dérivée. Mais sa formation, elle, appartient à l'histoire, et nous connaissons son milieu d'émergence : la Cité grecque. Les récits édifiants de ce miracle, avec son martyrologe, ne manquent pas : hagiographies des saints laïques luttant contre les forces obscures de l'archaïsme.

15 Mais cette manière de ménager des évidences n'est après tout que l'effet d'un procédé d'éclairage, - pédagogie républicaine ou génie, ou étiologie de l'homme de science. Car le foyer grec de la vérité de constat, ce lieu de lent basculement où l'efficacité singulière de la parole magico-religieuse cède devant l'évidence commune des preuves ne réside ni dans les plis de cerveaux géniaux, ni dans les textes savants que nos disciplines nous appris à vénérer. Il s'est formé dans un domaine de pratiques somme toute moins glorieux, mais très considérable : celui du règlement des litiges, et singulièrement des litiges entre inégaux. Athènes, cité des plaideurs.

Cette longue durée de l'agonie des ordres de vérités, - des manières de vaincre les incrédulités - on pourrait la baliser, presque pas à pas, à travers l'évolution des pratiques judiciaires et des séditions (stasis) grecques². Mais il est possible d'emprunter une perspective raccourcie: passé les Euménides d'Eschyle, les tragiques grecs ne cessent de la mettre en scène pour s'attirer les suffrages de la Cité.

Oedipe - roi, Foucault l'a montré maintes fois ${ }^{3}$, est de part en part dramatisation de ce processus d'invention judiciaire destiné à " juguler les puissants en fonction de leurs actes » : terrassement de l'affrontement ordalique des discordes par la confrontation jugée des valeurs, au terme d'une nouvelle ligne de partage entre le vrai et le faux qui passe par un procédé de verbalisation de la preuve des faits accomplis, et de mesure des forces politiques; exposition de cette technique d'administration du crédit (pistis) ${ }^{4}$ dont les règles permettent de retourner la mémoire du plus faible contre le dire efficace du plus puissant. Le témoignage d'un esclave ${ }^{5}$, confirmé par les voix du choeur ${ }^{6}$, y transforme la vertu hérö̈que du tyran-juge Oedipe en démesure, la démesure en méfait, et le méfait en cause assignable d'un fléau qui affecte la Cité toute entière, bref en culpabilité politique condamnable. Oedipe convaincu.

Cette procédure politico-judiciaire de recueil contradictoire des témoignages en vue d'un vote, qui donne forme aux savoirs grecs, la tradition des légistes lui a donné un nom : l'enquête. Elle n'est ni de tous les temps, ni de tous les lieux. Sa fonction et son optique varient à partir de stratifications successives : rétablir des proportions, centraliser des unités, composer des disparités, mobiliser des solidarités, contrôler des variations...

\section{Un problème fin de siècle: arrière-plans.}

19 Une telle lecture du " complexe» d'Oedipe, noeud d'un " pouvoir/savoir », il faut pourtant le dire, est d'abord la nôtre. Ces noces de la politique et de la vérité, sur l'autel du procès pénal, commentateurs et exégètes les ont peu célébrées jusqu'à une date 
récente. Et pour cause. Les enchaînements, les traitements transversaux qu'elle requiert supposent la constitution d'un corpus: la recherche, la collecte, la centralisation d'archives sur les pratiques judiciaires et les institutions politiques grecques. Et sans doute la constitution d'un corpus est-elle indissociable de conditions de possibilités (formation d'un milieu savant, avec son appareil et ses règles d'accès à l'information; mise au point de techniques de localisation, d'identification, d'enregistrement, de mobilisation, de classement, de combinaison des archives). Mais il est aussi indissociable de conditions de réalité: de la formulation d'un problème.

Or ce problème - celui des rapports entre règles de véridiction, formation du droit criminel, et changements des modes d'exercice du pouvoir lisibles à travers les «constitutions» (politéia) - est énoncé par le XIX siècle finissant, au moment même où émerge le journalisme d'investigation.

21 Il l'est dans le cadre d'une crise générale du droit, comme manière d'objectiver et de gouverner la société, les sujets, l'homme. Crise du libéralisme, - qui est d'abord pensée critique du droit, pensée de la liberté face à l'Etat de Police - liée aux effets de l'industrialisation, comme réalité engendrée par la politique libérale. Crise qui a partie liée avec la naissance des sciences sociales, et qui court tout au long des deux derniers tiers du siècle, avant d'éclater, de s'exposer au diagnostic, vers 1880.

Cette crise on peut en analyser les composantes à travers deux grands processus, dont je ne peux ici qu'évouquer le point de départ: la transformation de la vérité judiciaire, et celle de la criminalité autour de $1760^{7}$ constituent, je pense, la conjoncture et le contexte d'arrière-plan du journalisme d'investigation. Sur le fond de cette grande mutation on peut saisir sa formation à travers trois grandes étapes.

\section{I - L'apprentissage de la vérité d'instruction et de la procédure pénale (1820-1840).}

Dans la première moitié du $\mathrm{XIX}^{\mathrm{e}}$ siècle, le redéploiement des théatres du crime et des répertoires qui le verbalisent - ou pour le dire autrement, la redistribution des sources enclenche une lente mutation du récit de crime. Il prend forme sur de nouveaux registres. La nuée anonyme de placards, pamphlets, almanachs, littérature bleue qui papillonne autour des grands criminels s'étiole et disparait au profit du journal et du roman. On explique parfois ce transfert par les effets économiques de la liberté d'imprimer, accompagnée de la formation de réseaux de distribution, qui tarissent les circuits de l'édition illicite et détruisent ceux du colportage. Sans doute la formation d'un marché de masse des nouvelles, avec ses techniques de contrôle, joue-t-elle un rôle dans la substitution des supports. Mais le réagencement des récits de crime qui s'opère simultanément est avant tout l'effet de surface d'une triple mutation de la dramaturgie criminelle: pénalité, publicité, instruction.

24 - Mutation de la pénalité: lente extinction à partir de 1790, puis suppression légale vers 1840, des cérémonies de l'exécution publique, au profit du fourgon cellulaire opaque qui véhicule le condamné du tribunal à la prison ou au bagne. Avec ce moment de gloire s'évanouissent à la fois des marchés épisodiques, et le lieu de verbalisation de genres: ballades de pendus, sermons d'échafaud, confessions de pilori, derniers mots d'un condamné. La compilation rétroactive du Newgate Calendar, commencée en 1773, est le chant du cygne de ces cérémonies de la mémoire. Apparaît, en revanche un nouveau type 
d'archiviste régulier des vies infâmes: greffier ou visiteur de prison, compulsant des dossiers individuels, ou confessant des âmes perdues.

- Mutation de la publicité judiciaire: l'ouverture du procès criminel au jury et au public déplace le lieu de manifestation de la vérité judiciaire. Ce n'est plus seulement la vérité dérivée du juridict (de la sentence), que la justice offre au regard. C'est la cérémonie secrète du verdict, conjugant la clarté de l'administration des preuves aux ressorts confus de l'intime conviction, qui s'expose dans les règles à un public où se côtoient nouvellistes et écrivains. Sur les traces de John Wight, du Morning Herald de Londres ${ }^{8}$, des journaux vont se spécialiser dans cette chronique des véridictions infâmantes ${ }^{9}$. Sous la forme du compte rendu d'audience, le fait divers criminel y gagne en conformité ce qu'il perd en éclat singulier et entre en relation avec la routine accablante des instances correctionnelles, qui assurent continuité et hiérarchie des délinquances.

- Mutation de l'instruction: La figure du policier-détective, incarnée par Vidocq dès 1810, se propage dès les années 1830 sur toute l'Europe et jusqu'au Etats Unis ${ }^{10}$. La vieille relation du nouvelliste au renseignement policier s'en trouve changée. Ce que le reporter découvre au commissariat, c'est bien sûr un poste et des instruments d'observation des mouvements des bas-fonds: la mise en forme de faits incriminés sur les registres de plaintes côtoyant les incidents minuscules répertoriés sur les variétés de mains courantes. Mais ce sont aussi les actes et la scène de la vérité policière, ces routines codifiées de l'instruction et de l'information qui forment les coulisses du procès. Le crime n'est plus seulement scène à reconstituer sur les tréteaux du tribunal. Il est aussi, d'abord, mystère à dénouer, duel d'intelligences, poursuite d'une vérité d'instruction obéissant à des séquences d'actes et de transcriptions réglés.

New York - Il avril 1836 : Notre cité a été déshonorée ce dimanche par un des meurtres les plus insensés et prémédités que nous ayons jamais eu à rapporter.

Voici les circonstances, vérifiées sur les lieux mêmes ...

Commence avec le traitement de l'affaire Jewett-Robinson par le Herald de Bennett, l'appropriation de la procédure d'enquête par le journalisme. Enquête « parallèle ", qui épouse la temporalité de l'instruction et du procès, inaugurant un long et monotone chapitre de chamailleries dans l'histoire des relations entre presse, police et justice.

Commence, aussi, l'histoire du roman policier: Le Mystère de Marie Roget, qui se réfère directement à une affaire couverte par le Hera/d, date de 1842.

\section{II - Registres d'enquête: criminalité et société (1840-1890).}

On sait comment les techniques policières de l'enquête, manière d'établir des méfaits et d'imputer des responsabilités -, se propagent à partir des années 1840 sur les registres de l'accident. Le reportage y trouve de nouvelles règles de consistance ${ }^{11}$, et le fait divers, avec son art de l'ellipse ${ }^{12}$, un principe d'expansion, appuyé sur un journalisme de "tournée ». Le libéralisme y puise quotidiennement matière à sa grande pédagogie de la prévoyance.

Mais simultanément, rentrant en relation avec le calcul des probalités sur les tables des actuaires, l'accident revêt une forme qui déclenche l'irréversible crise de la responsabilité libérale et des institutions philantrophiques. Forme de l'occurrence statistique, qui fait basculer la tératologie délictueuse des libertés imprévoyantes dans les marges prévisibles de solidarités modulaires. 
31 L'accident cesse d'être chance dommageable, mal naturel contre lequel chacun a le devoir de se prémunir. Il devient risque collectif, mal social inhérent au bien commun, qui requiert prévention et assurance mutuelle. Le schéma causal de la responsabilité individuelle, qui fonde la régulation libérale et autorise la construction des faits, s'en trouve irrémédiablement fêlé. Dénouant l'intrication du dommage subi et de la faute commise, la rationalité du risque mine la règle d'imputation qui sous-tend la juridiction, le dire juridique.

32 L'introduction des circonstances atténuantes (1824, puis 1832 en France) marque ce moment d'ébranlement pénal de la causalité juridique. Lui répond, simultanément, la naissance d'une écologie de la pauvreté, adjoignant aux politiques de moralisation des pauvres les premières mesures portant sur leurs conditions d'existence. Mais ces aménagements ne suffisent pas à absorber le séisme qui disloque la pensée libérale. Corrélativement, la rationalité du risque enclenche en effet un nouveau jeu de véridiction, jeu d'immanence qui redéploie les faits observables et formulables dans deux directions: vers l'identification probabilitaire et le dépistage des individualités ; vers les principes fonctionnels de la vie sociale.

Dès les années 1840, avec une intensité qui ne cessera de croître, le traitement des pratiques criminelles s'engage dans cette bifurcation. La relation pondérée entre méfait et acte d'un malfaiteur se relâche au profit de nouvelles variations : sur les virtualités de l'individu malfaisant ou déviant, sur les relations entre méfaits récurrents et causalité sociale. Déplacement du champ d'observation savant, de surveillance policière, d'enquête journalistique.

34 a) sur le versant de «l'individu dangereux ", deux thématiques, qui sont aussi deux manières de gérer le risque criminel, de produire et d'enchaîner des faits remarquables:

- la thématique du criminel-né, avec sa muséographie positiviste des stigmates physiques héréditaires et son paradigme du crime contre-nature. Dans les dernières années du XIX siècle, Lombroso, Ferri et l'école italienne d'anthropologie criminelle lui donneront consistance mesurable, couronnant une lignée commencée avec Gall et Pinel, et jalonnée de médecins aliénistes (Pritchard, Lauvergne, Lucas, Morel, Despine, Maudsley...). Zola lui apporte la consécration littéraire jusque dans les colonnes de l'Estafette à l'occasion de l'affaire Gouffé13 ${ }^{13}$ et Bertillon la reconnaissance policière.

36 Contrepoint ironique à cette modification d'éclairage: le retournement de l'individualité délinquante en instrument de détection des inconduites dissimulées. Intempérance, licence sexuelle, goût du jeu, mensonges deviennent autant de stigmates disqualifiants que les reporters - surtout américains - iront traquer jusque dans les salons des puissants. Arrachée à l'amoncellement pénitenciaire des vies minuscules, la signalétique du vice trace une carte d'invasion de la vie privée des grands.

37 - La thématique de la dangerosité sociale, qui s'élabore autour du crime sans motif, avec son relativisme de la norme, son respect fonctionnel des garanties juridiques, et sa façon de traiter le méfait comme une occasion de punir un individu pour ce qu'il est (antisocial). Grande et terrible pensée de la Défense sociale, qui fait son deuil de la faute dans la responsabilité, de la liberté dans l'imputation, de la vérité au profit de l'efficacité. L'illustreront, au début du XX $\mathrm{XX}^{\mathrm{e}}$ siècle, sur les pas du juge d'instruction Tarde, le belge Prins, l'allemand Von Liszt, le hollandais Van Hamel, le suisse Stoos ou le norvégien Getz.

38 b) Autre versant, celui de la responsabilité collective. On y lie les occurrences et les fréquences du crime aux principes de l'organisation sociale. Ceux-là deviennent un effet 
dérivé de celle-ci, un taux de risque produit par une configuration de milieu, comme Bennett le concluait déjà dans les termes de l'hygiénisme :

«la mort d'Ellen Jewett est le résultat naturel d'un état de la société et de la morale qu'il faudrait réformer... elle découle aussi naturellement de notre culpabilité et de notre corruption générale que la pestilence des eaux de mort stagnant sous le soleil d'août».

C'est dans ce contexte qu'à la vague d'enquêtes sur le paupérisme qui déferlent sur les sociétés occidentales s'ajuste l'essor du «contre fait-divers» dans la presse populiste, réformiste, et les journaux ouvriers ${ }^{14}$. Il oppose l'indulgence dont bénéficient affameurs et patrons irresponsables à la misère criminogène que produisent leurs pratiques.

Si l'on s'en tient à ces grands traits évolutifs de l'aire "occidentale », les règles de formation du journalisme d'enquête, phasées avec l'évolution de la vérité judiciaire, ne divergent guère d'un pays à l'autre. Pourtant, c'est sur ce socle commun que se produit, me semble-t-il, une rupture.

41 Le journalisme d'enquête continental, dans son immense majorité, se cantonne à ce point sur ces acquis techniques et sur le territoire d'infraction produit continûment par le système judiciaire et l'appareil gouvernemental. Le " police-reporter» américain, lui, développe sur ces bases un registre d'information qui va faire de la presse une pièce constitutive du processus politico-judiciaire.

\section{III - L'émergence des «fouille-merde» (1880-1914).}

Inscrit dans cette lignée de l'enquête policière appliquée aux maux sociaux, le journalisme d'investigation américain est porté sur les fonds baptismaux le 14 avril 1906. L'imposition du nom est administrée par Théodore Roosevelt en personne, dans une philippique fameuse contre un type de journaliste assimilé à « l'homme au fouille-merde »(muck-racke) :

«l'homme qui ne pouvait regarder ailleurs qu'en bas, son fouillemerde à la main, auquel on offrit une couronne céleste contre son fouille-merde, et qui, sans lever les yeux, ni regarder la couronne offerte, continua à fouiller les excréments à terre ${ }^{15}$ ».

Il n'est évidemment pas question ici de retracer l'histoire des fouille-merde ${ }^{16}$, ni même de décrire une technique d'exposition. Le trait distinctif du «muckraking» ne réside en effet sans doute pas dans son procédé d'exposition des faits, conforme aux règles judiciaires d'administration des preuves. Il relève plutôt de sa façon de solidariser les méfaits, d'emboîter les illégalismes dans une causalité vitaliste. Les pratiques illicites qu'il poursuit ne revêtent ni la forme de l'infraction exorbitante, ni celle de l'écart à la norme exposant la dangerosité d'un sujet ou l'immanence du mal social. Elles ont la monotonie régulière d'un ajustement permanent des processus vitaux à l'entropie qu'ils produisent. La vieille figure du mal faire, que la justice s'obstinerait à corriger, devient avec eux le principe même de survie de l'organisme social: un principe impersonnel de régulation qui traverse la justice elle même.

Cette manière d'objectiver un enchaînement des illégalismes, isomorphe à un mode d'exercice du pouvoir, il me semble que l'on peut en saisir la formation à la jointure entre deux processus d'organisation : la conduite locale du développement urbain, qui met en évidence le fonctionnement des «machines» politiques ; la concentration industrielle, qui rend visible le contrôle des trusts (puis des holdings) sur l'économie nationale. Ce point 
de jonction, les hebdomadaires nationaux de la Réforme ${ }^{17}$ l'effectuent à partir des quotidiens urbains du «nouveau journalisme».

\section{1) La dégénérescence urbaine.}

Dès les années 1880 , l'interaction des composantes de la vie urbaine, régulée par l'appareil politique local, constituent l'objet principal du journalisme «indépendant» symbolisé par le PostDispatch (St Louis), puis le World (New York) de Pulitzer. Les sciences de la vie - la physiologie, le darwinisme et sa variante spencerienne - dessinent l'espace temps de cette lecture. Quant au plan de formation des évidences auxquelles elle s'applique, il suffit ici d'en rappeler sommairement quelques traits: explosion démographique, nourrie des déferlantes de l'immigration; repli local de la politique partisane après la guerre de Sécession; désenclavement des quartiers par la voirie et les transports, et prolifération d'agglomérations. Dans les vingt dernières années du siècle, la ville américaine est un opéré industriel permanent, éventré d'immeubles de rapport, perforé de conduites, quadrillé de rails, ligaturé dans les câbles. Maîtres d'oeuvre de ces corrélations: les vielles «machines» municipales, contrôlant des élus qui attribuent les marchés publics. Nouveaux registres de «mystères» pour Pulitzer et ses pairs.

Dans leurs colonnes, l'énigme du crime d'exception, la liturgie judiciaire du dénouement demeurent les matrices de criblage et d'exposition de l'information. Les accidents, leur prévisibilité, continuent à nourrir la nouvelle science du fait divers. Mais les grandes sensations, enclenchées par des campagnes où les pages éditoriales exposent les mobiles de l'information, viennent d'ailleurs: des enquêtes clandestines d'Elisabeth Cochran dans les asiles psychiatriques et les prisons, des descentes de reporters dans les cercles de jeu, les fumoirs d'opium, les maisons de passe. Ce que révèlent ces lieux opaques, protégés par la police, ce sont les voisinages entre le truand, l'entrepreneur et le politicien, la grande contagion de la corruption locale. Les fraudes fiscales des riches, les salaires des fonctionnaires municipaux entrent dans le champ de l'information, parce qu'ils sont des indices des transactions illicites qui enclenchent les maux urbains. Des conditions d'insécurité de l'habitat locatif, d'hygiène de la voirie urbaine, aux profits des entrepreneurs, et aux pots-de-vins qui circulent dans les assemblées élues, une même ligne de diagnostic: les bas fonds ne sont plus ces poches d'infection enkystées dans les quartiers délabrés. Ils se propagent sur le paysage urbain à la vitesse et dans les directions de l'organisation.

C'est sur ce fond populiste de criminalité épidémique qu'émerge la génération des " muckrakers ». Presque tous ont fait leur classe dans un quotidien au commissariat central d'une grande ville. Mais à Mulberry street, où il côtoie le vieux Jacob Riis, Lincoln Steffens n'a pas pour mission de couvrir les crimes. Ce sont les pratiques d'une police contestée qu'il est chargé d'observer ${ }^{18}$. Ce qu'il y apprend campe les règles d'un genre qui prendra ironiquement le nom de «journalisme d'investigation».

1. L'infraction visible n'est pas une transgression du code pénal. Elle est un franchissement de seuil à l'intérieur des pratiques illicites, un écart à la convention qui les règle. Ainsi du pickpocket qui prétendrait opérer hors de sa ligne de tram, ou du cambrioleur qui s'égare dans l'appartement d'un banquier.

2. Le mystère n'existe pas, et le protocole policier de l'enquête est un leurre. Le maillage catégoriel des populations délinquantes (voleurs, mendiants, homosexuels, prostituées, etc) est le dispositif d'éclairage de la police. Son contrôle suffit à faire la lumière sur tout fait incriminable. 
3. En revanche, le mystère est un procédé d'exposition, recourant aux techniques romanesques pour donner une forme juridiquement et véridiquement recevable à une manière de faire inavouable $\mathrm{e}^{19}$.

4. « Le métier de détective ne consiste pas à poursuivre, mais à former des amitiés avec les criminels". et au fouille-merde ${ }^{20}$. L'image du «muckraker» attablé entre le malfaiteur et l'homme de loi est un lieu commun des années 1900. Si elle vaut comme aphorisme, ce n'est pas pour les méchantes incriminations psychologiques auxquelles ses motivations donneraient ainsi prise. C'est pour le problème topologique que sous-tendent ces voisinages. Steffens le formule pour la première fois à Mulberry Street. Pourquoi la police doit-elle négocier des peines fictives - comme la fermeture temporaire des bars - avec les criminels qu'elle contrôle? Afin de sauvegarder ses revenus, prélevés sur les circuits du profit illicite (jeux, prostitution, etc...) qui alimentent la « machine ", martèle la presse populiste. Steffens forme l'image d'un autre plan de consistance, où voisinent les délits organisés et les processus vitaux de la finance :

«Comment la fermeture des bars peut-elle porter tort aux activités de Wall Street» demandais-je à James B. Dill, qui savait tout. Il me flanqua un coup de pied dans le tibia, fort, et après mon exclamation répondit à ma question: " pourquoi ta bouche crie-t- elle quand ton tibia a mal? » C'était la réponse, mais jamais je n'ai pu me satisfaire d'un fait ou d'une formule; c'était une image qu'il me fallait, un diagramme de la connexion entre les activités des bars et les banques, exactement comme j'en avais un du système nerveux qui reliait mes extrémités inférieures et supérieures. ${ }^{21}$.

\section{2) La mutation économique.}

«Jours Tweed à St Louis ${ }^{22}$. Dans son numéro d'octobre $1902^{23}$, le magazine McClure's lance la première série de l'ère des fouille-merde. La matière litigieuse traitée par Steffens est presque indiscernable des scandales affectionnés par les quotidiens sensasionnalistes : description des tractations de couloirs où la " machine» attribue les marchés publics, tarifs des élus etc... Mais ce qui change, avec les muckrakers, c'est l'espace-temps qui trame le mal faire dans une véridiction, le plan de consistance des pratiques illicites.

Comme les circuits de distribution de McClure 's, la corruption urbaine s'inscrit dans un schéma national ${ }^{24}$. Si les quotidiens urbains perçoivent si mal l'actualité, la rabattant sur une perspective louche, c'est que leur échelle d'observation est beaucoup trop grande. S'ils en médisent, multipliant les réquisitoires contre la politique et faisant le lit des dictateurs en herbe, c'est que leur organicisme entêté rend illisible le grand diagramme $\mathrm{du}$ "gouvernement invisible ». Les mouvements d'organisation qui incorporent les villes, ravageant les régulations locales, ne sont visualisables qu'à petite échelle, à même cette carte des transports ferroviaires qui accélère les vitesses en concentrant les capitaux. Les lignes de corruption qui en épousent les directions et les foyers ne sont pas les symptômes de maladies locales: elles s'enchaînent régulièrement dans le grand processus que la vie économique. Derrière les boulevards du crime, ou plutôt les traversant et leur donnant forme actuelle: l'activité des grands trusts, conduisant une mutation accélérée de la sociétée 25 . "MONDE DES AFFAIRES EGALE TRAHISON, CORRUPTION EGALE REVOLUTION », conclut Steffens. 
51 Imaginer un diagramme du "gouvernement invisible ", et non pas pourchasser des évidences aménagées, détecter le plan d'un «système» d'éclairage ${ }^{26}$, et non pas éclaircir des mystères en vue: si technique d'enquête des fouille-merde il y a, il ne faut pas la chercher ailleurs que dans cette scénarisation ${ }^{27}$.Les ramifications du diagramme, les nervures du système exposent, d'un geste, le découpage et le montage de l'information: non seulement l'espace de répartition des archives incriminantes, - ces «frictions» corrélatives à une manière de gouverner hors champ - mais la façon de les enchaîner pour exposer cette manière.

Investigateur, je ne l'étais pas écrira Steffens. Je suis simplement allé là où d'autres avaient fait le travail, et cueillant les fruits de leur peine et de leurs risques, j'ai décrit et interprété leurs preuves ${ }^{28}$.

Ida Tarbelj ${ }^{29}$, Ray Stannard Baker ${ }^{30}$, Charles Edward Russell ${ }^{31}$, David Graham Phillips ${ }^{32}$, ces grands voyageurs du muckraking ne procèdent pas autrement que Steffens : ils testent des diagrammes, effectuent des trajets de montage entre des foyers d'accumulation des preuves. Double condition à cette vérification fragmentaire: le foisonnement des juridictions américaines, des instances d'accusation et d'enregistrement locales où les méfaits sont avérés sous la forme crédible du juridique; le maillage continental des chemins de fer et du télégraphe.

53 Etrange retournement du paysage véridique, dans ce moment d'apothéose de la vérité de constat. Ce que ne cessent d'éprouver les fouille-merde, c'est que la vérité a ses lieux privilégiés, mais aussi ses instants favorables: qu'il n'est de vérité que de circonstances, d'actualité.

54 L'actualité du muckraking, c'est sur le double versant de l'information concrète, et de l'information réelle, une conjoncture et un contexte produits par leurs déplacements. Conjoncture: l'articulation des vitesses du transport des matières premières ${ }^{33}$ à celles de la production ${ }^{34}$, de la distribution ${ }^{35}$, et de la consommation ${ }^{36}$. Articulation concrète, offrant prises aux complicités réticulaires entre concentrations industrielles verticales. Contexte: l'imbrication tactique des discours prenant comme objet les effets de cette conjoncture, pour problématiser une nouvelle manière de gouverner "vraiment ", de penser l'action gouvernementale à la vitesse et dans la transparence de l'événement. Un contexte qui maille Roosevelt, et sa régulation du Big Business par le Big Government, avec Upton Sinclair et Jack London et leur rêve de régulation socialiste. A l'intersection de la conjoncture et du contexte : un dispositif scripturaire, assurant prise sur et conformant les hommes, les choses et les énoncés.

Pour le dire autrement, le plan de consistance du muckraking, c'est celui de la formation de la «société de l'information », ou de ce que Gilles Deleuze appelle plus précisément «société de contrôle ». Ce plan qui s'effiloche, aujourd'hui, sous la poussée d'un nouveau diagramme ...

56 L'époque glorieuse des fouille-merde s'achève en 1914. Il Y a plusieurs explications à cette date: les faillites successives des magazines de la Réforme, où beaucoup lisent la main visible de la finance et de la publicité; la trahison des muckrakers eux-mêmes, engagés dans le militantisme socialiste, ou fondant la première machine de propagande gouvernementale du Comité Creel ; la guerre enfin, et ses priorités. On peut suggérer une hypothèse plus simple: les fouille-merde s'éteignent par exténuation, parce qu'ils ont dit tout ce qu'il y avait à dire, épuisé leur problème. Simultanément, un nouveau champ de gouvernement invisible émerge, appuyé, lui aussi, sur son dispositif scripturaire. Il est corrélatif à l'espace moderne de la guerre, investi par l'économie et la finance: 
international. Son premier fouille-merde est, au sens propre, l'élève de Steffens, qui l'a recruté personnellement à Harvard: Walter Lippmann.

\section{La justice des faits.}

Objectivation de l'exercice du pouvoir comme processus d'infraction, procédure de mise en diagramme des délits, cartophiant les faits bien formés, procédé juridico-policier de mise en récit des preuves enregistrées: trois composantes de la pierre de touche du journalisme d'investigation, dans ce grand moment d'invention. Il faut, cependant, y adjoindre une dernière épreuve de vérité, et non la moindre: la suspension de la vindicte morale, - du jugement -, au profit du diagnostic d'actualité. Il est certain que les articles des muckrakers participent au grand processus juridico-politique qui, dans cette période, incrimine et sanctionne des individus. Mais aucun fouille-merde digne de ce nom ne poursuit un but si médiocre. Leur conception de la causalité de leur interdit.

Car si la prolifération des injustices les affecte évidemment, dans ce moment de crise, ils y voient avant tout l'effet de surface de l'instauration de nouveaux rapports de justice, immanents à une transformation de la vie : non des dommages causés par des individus malfaisants, mais des effets impersonnels de causes naturelles, écrit Lincoln Steffens ${ }^{37}$.

Sans doute le journalisme d'investigation, aujourd'hui, ne peut-il échapper totalement à sa généalogie inquisitoire ou au noeud politique grec du véridique et du juridique qui forme le réel complexe d'Oedipe: la variété des juridictions lui imprime, d'emblée, les registres de preuves sur lesquels ourler ses exposés convaincants. Pauvre vérité, cependant, que cette méchante bordure intérieure, sur laquelle campent les chiens de garde de la diffamation. Vérité de procure, qui ne vaut que son rapport à l'extérieur, à cette enveloppe du présent que le fouille-merde, patiemment, parvenait à rendre visible à travers l'enchaînement des méfaits. Fouiller la merde peut être un beau métier, à condition de ne pas confondre l'intérieur et l'extérieur, de ne pas mélanger les tâches: non pas imputer des fautes, faire justice, mais plus innocemment, - et c'est plus difficile penser la justice des faits, l'actualité. 


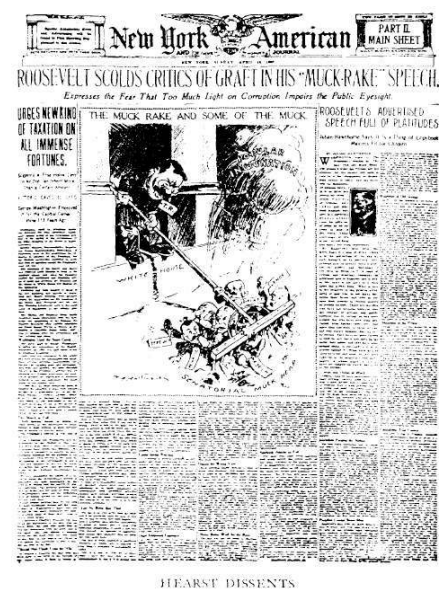

\section{BIBLIOGRAPHIE}

Brace and Cy, H., (1931), The AUfobiography of Lincoln Steffens, New York, t.I.

Cruikant, G., (1824), Morning at Bow street, Londres.

Fermigier, André, (1987), in Maupassant, La Petite Roque - Folio, Gallimard.

Filler, L., (1976), The Muckrakers, The pennsylvannia State University Press.

Foucault, Michel, (1983), « La maison des fous », in Basaglia, F., et Ongardo, F., Les criminels et la paix, PUF, pp. 145-160.

Leonard, C., (1986), Lettre de Sam McClure à Lincoln Steffens 20 janvier 1903 - citée in, The power of fhe press, Oxford University press, New York.

Sophocle, $(-430 ;-415)$, Oedipe-Roi.

\section{NOTES}

1. Sur cette distinction, cf Michel Foucault, "La maison des fous ", in Basaglia, F., et Ongardo, F., 1983, Les criminels et la paix, PUF, pp. 145-160.

2. Pour l'essentiel, ce cheminement a été accompli magistralement dans les oeuvres de Louis Gernet et Moses Finley. 
3. En particulier Michel Foucault, 1974, A verdade e as formas juridicas, Rio de janeiro - Cadernos da P. U.c.

4. Terme judiciaire consacré par la Rhétorique d'Aristote, et que Méderic Dufour et André Wertelle traduisent étrangement par \{( preuve ». (Les belles Lettres 1967 et 1989).

5. Sophocle, Oedipe-Roi, v. 1110-1185.

6. Ibidem v. 1186-1221.

7. Réforme de la procédure judiciaire et et du système pénal, division et dissymétrie des illégalismes, contrôle policier de la délinquance: sur ces points voir Foucault, M., 1975, Surveiller et punir, Gallimard.

8. Cruikant, G., Londres 1824, Morning at Bow street.

9. En France: La Gazette des Tribunaux ( $\backslash 825)$, le Courrier des Tribunaux, le Journal des Concierges (\836).

10. Après la police de la Tamise, confiée en $\backslash 798$ à Robert Colquhoun, la police de Londres voit le jour en 1829, celle de New York en 1844.

11. A commencer par cette règle du quis? quid ? ubi ? quibus auxiliis? cur? quomodo ? quando ? (qui? quoi? où ? avec quelles aides? pourquoi? comment? quand ?) quadrillant le fait judiciaire et ses circonstances, qui provient, via l'institution Oratoire de Quintilien, de la Rhétorique d'Aristote.

12. Cf la préface d'André Fermigier in Maupassant, La Petite Roque - Folio, Gallimard 1987.

13. Un couple infernal, Michel Eyraud et Gabrielle Bompard, a assassiné l'huissier Gouffé. Pour les juges, Eyraud est le véritable responsable, et Gabrielle n'est qu'une comparse. Une photo des protagonistes suffit à Lombroso pour flairer l'erreur judiciaire: Quoique les femmes coupables n'aient presque jamais la physionomie criminelle, Gabrielle Bompard, elle, l'a complètement ( .. .) (Le Gaulois, 20 décembre /89/). Zola renchérit trois jours plus tard: Gabrielle est certainement un type anormal. Elle a été nubile à huit ans, et, dès cet âge elle était complètement formée. Tout prouve qu'elle est très portée vers les plaisirs des sens; c'est une hystérique passionnelle, une malade, une dégénérée ( ... ).

14. En France, la Phalange, L 'Humanitaire, l'Atelier, Pauvre Jacques, etc ...

15. Cité in Filler, L., 1976, The Muckrakers, The pennsylvannia State University Press, p. 253. Le président Roosevelt extrait l'image de l'homme au fouille-merde du célèbre Pilgrim 's Progress de Bunyan.

16. La littérature américaine sur se sujet comporte plusieurs dizaines de titres. Cf en particulier $C$ Regier, c., 1932, The erea of muckrakers, University of North Carolina Press, M. Chalmers, D., 1964, The social and political ideas of muckrakers, New York, et les nombreux ouvrages de Louis Filler.

17. Principaux titres: McClure's, Collier's, The Arena, Success, The American magazine, Everybody's, Cosllopolitan.

18. Son affectation par l'Evening Post est liée à la campagne de la «Société pour la prévention du crime» menée contre la police de New York par le pasteur Parkhust. Nourrie par une armée de détectives et d'avocats, celleci débouche sur la grande enquête publique du Lexow Comittee en 1894, et sur la purge consécutive infligée par le gouverneur T. Roosevelt en 1898.

19. Vous savez que la méthode idéale d'écriture est celle qu'applique Alexandre Dumas dans ses grandes aventures de d'Artagnan, où périodiquement la narration atteignait un puissant point culminant. Cef article doit être arrangé de la même manière ... (Lettre de Sam McClure à Lincoln Steffens 20 janvier 1903 - citée in C. Leonard, T., 1986, The power of fhe press, Oxford University press, New York, p. 182. Matière incriminable, véracité judiciaire, suspense périodique, publication en feuilleton: la recette de la série policière.

20. En février 1901, McClure's commence la publication de la série de Josiah Flynt sur les bas fonds, The World of Graff (le monde de la combine). Elle deviendra une bible du muckraking qui utilise le terme «graft » comme nom commun des actes délicieux qu'il décrit.

21. Brace and Cy, H., 1931, The AUfobiography of Lincoln Steffens, New York, t.I, p. 220.

22. Titre choc: Tweed est la figure mythique du «boss» de la machine new-yorkaise. 
23. Theodore Roosevelt assure depuis un an l'intérim du président McKinley, mort des suites d'un attentat.

24. La série sur Saint Louis sera suivie de séries sur six autres villes, réunies en 1904 dans un livre: La honte des cités.

25. Muckraking signifiait à l'origine dénonciation de la friponnerie des corporations et des trusts, écrira Charles Edward Russel.

26. Gouvernement invisible et Le système sont deux formules fameuses de Steffens, devenues cris de ralliement des muckrakers.

27. Le « diagramme de Steffens» :

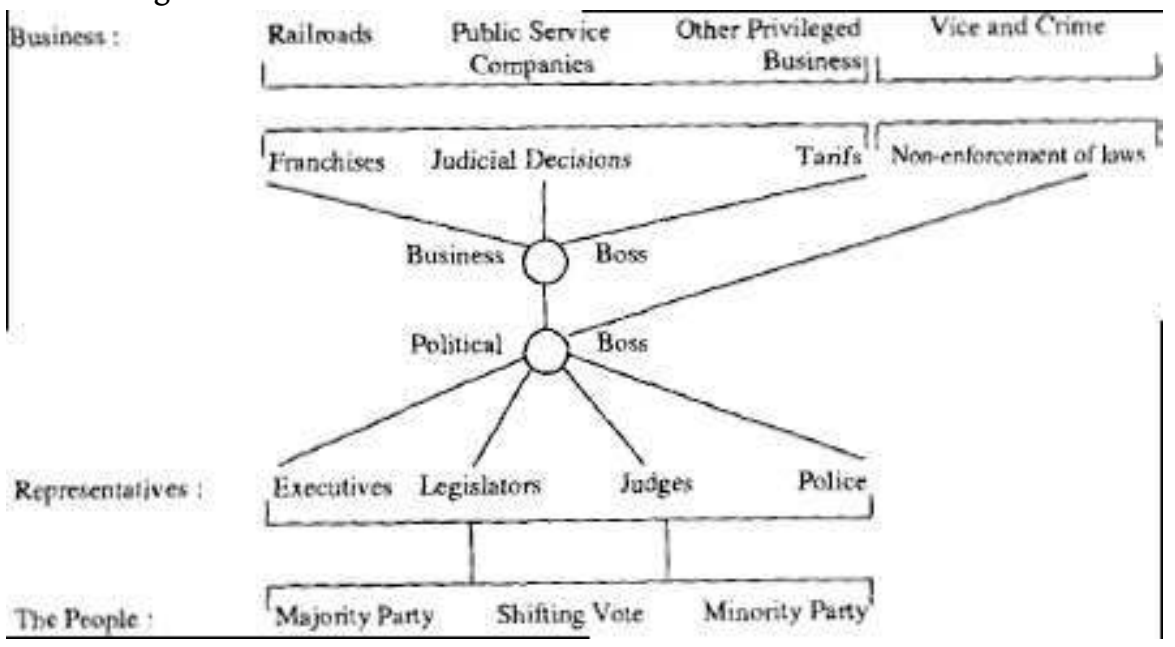

Ibidem t. II p. 596.

28. Lincoln Steffens - ibidem p. 216.

29. History of the Standard Oil Company (McClure's 1903).

30. The Right to Work, 1903 ; The Rai/road on Trial, 1905 etc ... (McClure's) ; Following the color line (The American Magazine - 1907).

31. The Greatest Trust of the World - sur le trust du boeuf - (Everybody's - 1905).

32. The Treason of the Senate (Cosmopolitan - 1906) serait à l'origine du discours de Roosevelt sur « l'homme au fouille-merde».

33. Le diagramme arborescent de McCallum, directeur de la compagnie de chemin de fer de l'Erie, qui vise à assurer le « gouvernement des voies» en temps continu par l'état major date de 1856.

34. Matérialisé dans l'architecture des aciéries Carnegie de Pittsburgh (1875) dessinées pour la circulation des wagons.

35. 1890: les compagnies de chemin de fer mettent en place la comptabilité des wagons, qui permettent de positionner quotidiennement les unités de chargement sur le réseau.

36. 1888 : naissance de Printers's Ink, organe professionnel de l'industrie publicitaire.

37. Ibidem, p. 403.

\section{RÉSUMÉS}

De l'invention de l'enquête criminelle à l'apparition des «muck-rakers » aux Etats-Unis, l'auteur entreprend une généalogie du journalisme d'investigation. Trois étapes sont analysées. 1820-1840 
: appropriation de la procédure d'enquête, liée à une mutation de la dramaturgie judiciaire et des récits qui l'accompagnent. 1840-1890: infléchissement des procédés de description des pratiques et des individus criminels, sous l'effet de la statistique. A la figure de l'individu d'exception se substitue celle du malfaiteur probable - criminel né, antisocial -; à la faute individuelle, la responsabilité collective de milieux. 1890-1920 : les "fouille-merde », associés au mouvement réformiste, déplacent le champ de l'enquête judiciaire en l'appliquant à la vie économique. L'auteur les caractérise par leurs objectifs, leur pratiques de «mise en diagramme» des méfaits d'un gouvernement invisible et leurs techniques de récit.

The author makes a research about the origines of journalistic inquiries. It first appeares in conditions similar to those of the old Continent : it had to go through an alteration in the formal proceedings of juridical cases ans in the stories which followed (1820-1840). Criminals are no longer seen as exceptional or monstrous characters, but with development of statistics, doctors as well as policemen and journalists try to delineate the factors of those who might possibly become criminals whether from their birth or from their surroundings. Scientist, police force and journalistic inquiries had altered their field of observation.

But muck-rakers only appeared in the U.S. (1890-1920). The author sets forth his specific aspects by making clear his aims, how he conducts his inquiries and the way he writes.

\section{INDEX}

Mots-clés : investigation, journalisme, presse, vérité

Keywords : investigation, journalism, press, truth 